\title{
The increasing importance of the biomechanics of impact trauma
}

\author{
MURRAY MACKAY \\ Mull House, Ballagarrett, Bride, Isle of Mann, IM7 BEA \\ e-mail: mmackay@netcomuk.co.uk
}

\begin{abstract}
The evolution of experimental biomechanics and crash injury research is summarized briefly to show that they both play a major role in mitigating traffic deaths and injuries. Historically, the subject has been based largely in western countries and thus focused on vehicle occupants, whereas some $80 \%$ of traffic casualties in the world are outside the vehicle as pedestrians, cyclists and motorcyclists. The subject is close to the regulatory process which controls vehicle design and is thus heavily influenced by government and industry, yet it is now in an expanding period because of new techniques to replicate the human frame's response to impact forces. New knowledge is likely to emerge from addressing population variations and combining real world accident investigations with experimental biomechanics. The application of impact biomechanics to the vulnerable road users is of particular importance.
\end{abstract}

Keywords. Biomechanics; population variations; accident investigation; injury criteria; vulnerable road users.

\section{Introduction}

\subsection{The early recognition of impact dynamics}

An implicit recognition of impact biomechanics exists in all of us and is engendered from an early age. Soft, deformable structures are benign, sharp, rigid objects like kitchen knives deserve caution. From the earliest of times the underlying principles of impact biomechanics have been recognized, in that implements designed to maximise trauma are hard, and concentrate loads such as spears and clubs, whilst conversely shields and armour absorb and distribute loads and protect the vulnerable parts of the anatomy. Hippocrates, writing around $400 \mathrm{BC}$, noted that for head injuries:

'Of those who are wounded in the parts about the bone, or in the bone itself, by a fall, he who falls from a very high place upon a very hard and blunt object is in most danger of sustaining a fracture and contusion of the bone, and of having it depressed from its natural position. Whereas he that falls upon more level ground, and upon a softer object, is likely to suffer less injury in the bone, or he may not be injured at all.'

Like most engineering subjects, impact biomechanics has evolved from early observations of natural phenomena, through an experimental period, to a theoretical framework which 
establishes general laws and precepts. Hugh DeHaven is credited with the first insights into human tolerance to crash forces. In 1917 as an young Air Cadet in Canada, he was involved in a mid-air collision. Whilst convalescing from his injuries, he realised that his survival was due to the maintenance of the integrity of his cockpit which, together with a safety harness, protected him from localised contacts and catastrophic injuries which killed the other pilot. He also observed that his own serious abdominal injuries related to the buckle of his harness, which localised the loading to cause a laceration of the liver. Crash-protective design, rather than capricious good fortune, had ensured his survival.

DeHaven (1942) analysed the circumstances of eight people who fell from considerable heights, seven of whom survived. Speeds of impact ranged from 60 to $95 \mathrm{~km} / \mathrm{h}$ and the objects struck consisted of fences, a wooden roof, soft ground and in two cases the bonnets of cars. From calculated impact speeds and stopping distances (the depths of the dents), decelerations were estimated and the groundwork for whole body tolerance to collision forces was laid. Subsequently Snyder et al (1977) developed this approach into a most useful methodology for obtaining such data.

In 1941, Sir Hugh Cairns published a paper on fatalities occurring among Army dispatch riders. He showed that for those who wore helmets the head injuries were relatively mild. Cairns (1946) showed that following the introduction of compulsory wearing of helmets by Army motorcyclists there was a progressive decline in the death rate. He also noted that most of the blows on helmets were to the front and side rather than to the crown. The consequences of these observations have been reflected in the evolution of the jetstyle and full-face helmets of today and the demise of the cradle suspension inside the helmet.

John Lane in Australia in 1942 noted that aircraft should be certified in two ways: they should be both 'airworthy' and 'crashworthy', and thus the term 'crashworthiness' was born. Its application to car design however, did not begin until some 20 years later.

The experimental period of biomechanics got under way after World War II, with studies by Gurdjian \& Lissner (1958) using cadavers to examine head injury in the main, and volunteer studies on whole body tolerance by Stapp (1951). The great contribution of Stapp was to show that the primary deceleration forces acting in the majority of car collisions are entirely survivable if the packaging of the human frame is satisfactory. He showed that accelerations of $30 \mathrm{~g}$ for up to $0.5 \mathrm{sec}$ were entirely tolerable, with only reversible soft tissue bruising occurring. At $45 \mathrm{~g}$ signs of mild concussion and retinal haemorrhage begin to show. These were decelerations measured on the seat of a dynamic sled. The accelerations experienced by the head itself were much greater.

In the 1950, Severy \& Mathewson (1954) were developing the techniques of experimental crash testing with instrumented dummies and high speed film analysis. By the mid-1960s a body of knowledge had developed that gave insights into the general frequencies of road traffic collisions and injuries, some understanding of the actual mechanisms that generate the injuries and some empirical means whereby the forces and accelerations to vehicle occupants could be modified. What was largely missing was accurate information on the tolerance of the various part of the human frame to the specific impact loads, and ideas of the likely benefits that might come from practical changes in vehicle design.

In parallel with the increasing amount of experimental work on both cadavers and animals, studies of real world trauma continued. An elegant paper by Sheldon (1960) entitled 'On the natural history of falls in old age' showed how the routine observations of a practicing clinician could lead to new insights into the aetiology and mechanisms of injury, particularly for long bone fractures in the elderly. 
Pioneering work on seat belts was conducted in Sweden where by 1960, some 50\% of private cars had belts fitted. The appropriate elongation and geometrical characteristics of belt systems were evaluated experimentally by Aldman (1962), who demonstrated the importance of correct anatomical positioning and the dynamic properties appropriate to the deformation and geometrical dimensions of the interiors of specific car designs.

The subject of biomechanics in relation to car occupant protection grew rapidly in the 1950s and 1960s, and became institutionalized with the establishment of the National Highway Safety Bureau in 1966 by an Act of the US Congress. That agency initiated a set of standards controlling the performance of cars in terms of their crashworthiness. Those standards have reverberated throughout the automotive world ever since. They have been copied, modified and adopted by almost every country with a significant car population. They changed car design from being a free market activity, largely dominated by styling, to one in which certification, or passing the standards, or more recently getting good grades or stars in the various consumer information tests, is of prime importance in the priorities of the car manufacturers.

The scientific basis of those first generation crash performance standards was not well founded; many of the requirements were informed guesswork only. With the benefit of hindsight, quite a few major mistakes were made, but what has become clear is that the subject is much more complex than the knowledge of the 1960s assumed. The real world of collisions contains many surprises. Common sense has that most curious property of being more correct retrospectively than prospectively. A major gap was left as the subject became more under the control of government and industry and away from the individual efforts of the early workers.

The problem was evaluation. Consider a simple example, early field accident studies had shown that, contrary to the common sense of the times, it was better statistically to remain in a car during a collision than to be thrown out. Most people were thrown out because doors opened during the crash, and the concept of an anti-burst door latch developed. Thus a problem was identified, a solution proposed, and the legislative machinery went into action. Standards were written specifying the longitudinal and lateral strengths of door latches, specified in isolation from the body shell in to which they were to be mounted. The car industry, virtually worldwide, redesigned car door latches to meet the new requirements, the new cars were built and sold, and the problem of ejection was then supposed to go away. It was left to individual workers like Huelke et al (1963) and Gissane \& Bull (1962) to actually look at the real world of collisions and attempt to find out if these new regulations were in fact producing the benefits for which they were conceived. Systematic feedback into the legislative process did not exist then and to some extent still does not exist.

A parallel can be drawn with the drug industry. Before a new drug is marketed, it is subject to rigorous trials, its performance is monitored for effectiveness and possible side effects. In contrast, the introduction of crash protective vehicle design measures was, and still is, relatively haphazard. Yet, redesigning the front structures of cars to produce less injury to pedestrians and cyclists, or introducing airbags into cars, is a public health measure every bit as important as a new controlled drug.

In the case of anti-burst door latch design, evaluation studies showed that door opening rates in collisions were reduced from $30 \%$ to $15 \%$, and deaths were reduced by $5 \%$. But doors still continued to open in collisions and detailed studies by Mackay (1969) showed that they did so in the real world in ways not covered by the regulations, largely because of the manner in which the door latch and striker were mounted in the body shell of the car and stressed under actual impact conditions.

Haddon (1968) proposed a rational approach to the analysis of traffic accidents and the counter-measures to be applied. He drew a parallel with the classical public health definitions 
of 'host', 'agent' and 'environment' as the 'road user', 'the vehicle' and 'the highway', and applied them to the 'pre-crash', 'crash' and 'post-crash' phases of an accident. Of the nine cells in that matrix, the central one of the crash phase and the vehicle covers the biomechanics of impacts and the crashworthiness of vehicles. By institutionalizing crashworthiness through regulations, Haddon, as the first director of the National Highway Safety Bureau, helped to generate two important areas of biomechanical research; experimental biomechanics and studies of the epidemiology of road injuries.

\section{The rise of experimental impact biomechanics}

The 1980s and 1990s saw a major increase in experimental impact biomechanics. The main advances were in three areas. First, the experimental use of biological material, animals and cadavers, led to a much better understanding of the mechanisms of specific injuries. For example, the response of the chest to an anterior blunt impact initially was thought to be assessed adequately by measuring the peak acceleration at a posterior position on the thoracic spine. Research showed however that the very viscous response of the chest makes it sensitive to rates of loading and the extent of the compression of the rib cage. Thus alternatives to acceleration were developed based on chest compression and the velocity of the anterior chest wall, first described as the 'viscous criterion' by Viano \& Lau (1985).

Similarly for head injuries, the early work of Gurdjian which led to the development of a relationship between head injury risk and the linear acceleration of the head measured at its centre of gravity, was shown to be an inadequate parameter, particularly for certain types of brain injury. Diffuse axonal injuries in particular were shown by Thibault \& Gennarelli (1985) to be related to angular accelerations and durations rather than linear accelerations.

The focus of much of that period of experimental biomechanics was to produce Injury Criteria: to come to a decision on what is an acceptable level of load applied, for example, to the femur axially through a knee impact with the instrument panel of a car, or the acceptable levels of head acceleration and duration when the head strikes a steering wheel, a windscreen or the side roof structure. Such single point criteria are still the bedrock of crashworthiness design today, and are used to assess the crashworthiness of vehicles by governments and consumers around the world.

The second major area of experimental biomechanics in the 1980s and 1990s was in the development of human surrogates or dummies. The aim was to replicate the human frame with sufficient biofidelity so that, with instrumentation mounted in the head, the chest, the femurs and elsewhere in a dummy, the readings obtained when the dummy was placed in a car and crashed under carefully controlled conditions would assess the actual exposure to injury of the average male human, using the injury criteria developed from cadaver and animal studies.

Such an approach assumes that a physical dummy, made of steel and plastics, can replicate the response of the biological materials which make up the human frame. A large amount of research effort has been put into producing and validating better dummies. Different crash conditions require different dummies. Side impacts present different requirement from frontal impacts; low speed rear impacts require special thoracic and cervical spines. Population issues are in part addressed with $5^{\text {th }}$ percentile female and $95^{\text {th }}$ percentile male dummies, plus an expanding family of child dummies from the new born infant to the 10 year old child. An acceptable pedestrian dummy has yet to be developed but much effort is currently going into such a device.

All of this work on developing injury criteria and measuring the response of dummies in certain specified crashes, and hence the ability of a car's crashworthiness to protect its 
occupants, is based on the assumption that those procedures represent the populations of real people in the real world of road accidents.

The third thrust of biomechanical research in the last three decades has thus been to develop the epidemiology of crash injuries, the nature and frequency of the various crash types, their severities and outcomes on the actual human population at risk. Only then can the value of good crashworthiness design in the real world be assessed.

\section{Field accident research - the broad picture}

Currently about 1.2 million people die in road crashes each year. That number will rise to over 2 million by $2020.85 \%$ of these crashes occur in developing countries and of those about $75 \%$ are pedestrians, cyclists and motorcyclists. Beyond fatalities, data on traffic crashes and the consequent injuries to survivors on a global scale are fragmentary, but over the last ten years several organizations, notably the World Health Organisation (2004), have attempted to outline the nature and severity of these traffic injuries and predict trends for the next decade. Such efforts have been part of a wider effort to quantify the health of the world's population.

Under-reporting is a major factor. Even in highly motorized countries with relatively complex data collection procedures, certain classes of traffic casualty are significantly underreported. In the EU for example cyclist, pedestrian and motorcyclist casualties treated in hospital are not recorded in police data bases by amounts which vary from $10 \%$ to $60 \%$ depending on injury severity and class of road user (Mackay 2005). In many low income countries without adequate recording procedures, the majority of traffic casualties go unrecorded. Hence, projections from sample studies are necessary to attempt quantitative assessments, with all the associated uncertainties. Even for fatalities under-reporting in many countries is significant. With these caveats, however the relative importance of traffic crashes today and over the next fifteen years can be described.

In 1998 the World Health Organisation estimated 1,170,694 road traffic deaths worldwide. Such deaths were the tenth leading cause of death for all ages, accounting for $2.2 \%$ of global mortality. Road traffic deaths were the second leading cause of death for those aged 15-44 years (22 deaths per 100,000) and the third leading cause of death for the 5-14 year age group (14 deaths per 100,000) (Krug 2000).

Traffic crashes accounted for 1,029,037 deaths in low- and middle-income countries ( $88 \%$ of global mortality from crashes) and 141,656 deaths in high-income countries (OECD grouping), or only $12 \%$.

In economic terms, Jacobs et al (2000) have shown that the traffic crashes absorb about $1 \%$ of GNP in less motorized countries, $1.5 \%$ in transitional countries and $2 \%$ in highly motorized countries, although within each group there are significant variations from $0.3 \%$ in Vietnam to almost 5\% in the USA, and Kwa Zulu, Natal. For the United Kingdom the annual cost approaches $2 \%$ of GNP as it does for the EU as a whole.

The diverse nature of traffic crashes between the major classes of traffic death in a selection of countries is shown in table 1 .

Even from this small selection of countries, it is clear that in the developing world the very predominant classes of casualties are the vulnerable road users - pedestrians, cyclists and motorcyclists. Vehicle occupants are a minority of the fatalities (and casualties) in those countries. What also appears, however, is that even in highly motorized countries such as Japan and the EU, the vulnerable road users are important. In Japan they are the majority of fatalities, and in the EU they represent about a quarter. Indeed the United States is the unusual motorized country in having a large majority of its fatalities as vehicle occupants. 
Table 1. Road user fatalities as a percentage of all fatalities.

\begin{tabular}{lccccc}
\hline Country/city & Pedestrian & Bicyclist & MTWV & Cars/LTVs & Buses, etc. \\
\hline Thailand & $47 \%$ & $6 \%$ & $36 \%$ & $12 \%$ & - \\
Malaysia & $15 \%$ & $6 \%$ & $57 \%$ & $19 \%$ & $3 \%$ \\
Delhi & $42 \%$ & $14 \%$ & $27 \%$ & $12 \%$ & $5 \%$ \\
Japan & $27 \%$ & $10 \%$ & $20 \%$ & $42 \%$ & $1 \%$ \\
Netherlands & $10 \%$ & $22 \%$ & $12 \%$ & $55 \%$ & - \\
Australia & $18 \%$ & $4 \%$ & $11 \%$ & $65 \%$ & $2 \%$ \\
EU (15\%) & $16 \%$ & $5 \%$ & $16 \%$ & $57 \%$ & $6 \%$ \\
USA & $13 \%$ & $2 \%$ & $5 \%$ & $79 \%$ & $1 \%$ \\
\hline
\end{tabular}

Almost everywhere, national traffic accident data are collected by the police. By their nature such data bases contain little biomechanical information but they do give a broad numerical picture of road user classes, types of collisions and general injury outcomes, even if poorly defined. However, the need to have better information about crash severities, the origins of specific injuries, the variations which occur throughout the populations at risk led in the early 1980s to a growth in detailed sample studies of specific types of accidents where researchers actually investigate the accidents themselves, often at the scene (Mackay et al 1985). Such techniques are labour intensive, but important and have given rise to much new biomechanical knowledge of the last two decades.

\section{In-depth crash injury research}

More detailed accident investigation data bases are the main source of our epidemiological knowledge. By providing such knowledge, priorities for counter-measures can be set, regulatory tests can be justified, and the benefits and limitations of improved crashworthiness of vehicles can be evaluated. At a more detailed level, specific mechanisms of injury are described and their frequency and consequences assessed. Just as an example, detailed crash investigation studies of US Indy car crashes, where the cars are fitted with comprehensive crash recorders, have suggested that the current thoracic injury criteria may be overly conservative, although the population of crashes in that sample is not typical in that it is restricted to very fit, young males (Melvin et al 1998).

Many current studies link field accident data with experimental biomechanics. Studies of the brain's greater susceptibility to diffuse injury when struck laterally rather than frontally have been confirmed in detailed accident investigation studies. Head and neck injuries to small females from airbags gave an impetus to the development of the current out of position test requirements for airbag design to meet. Detailed accident investigation is a way of examining the biofidelity of our current dummies and modelling techniques. By comparing the kinematics of real people in real collisions with that of dummies in comparable collisions, the limitations of our current test tools can be examined. These are just a few examples of how detailed field accident investigations contribute to biomechanical knowledge.

\section{Current issues}

Of particular interest currently is the area of population variations. Historically, vehicle crash performance has been specified by requiring that certain parameters measured in a given 


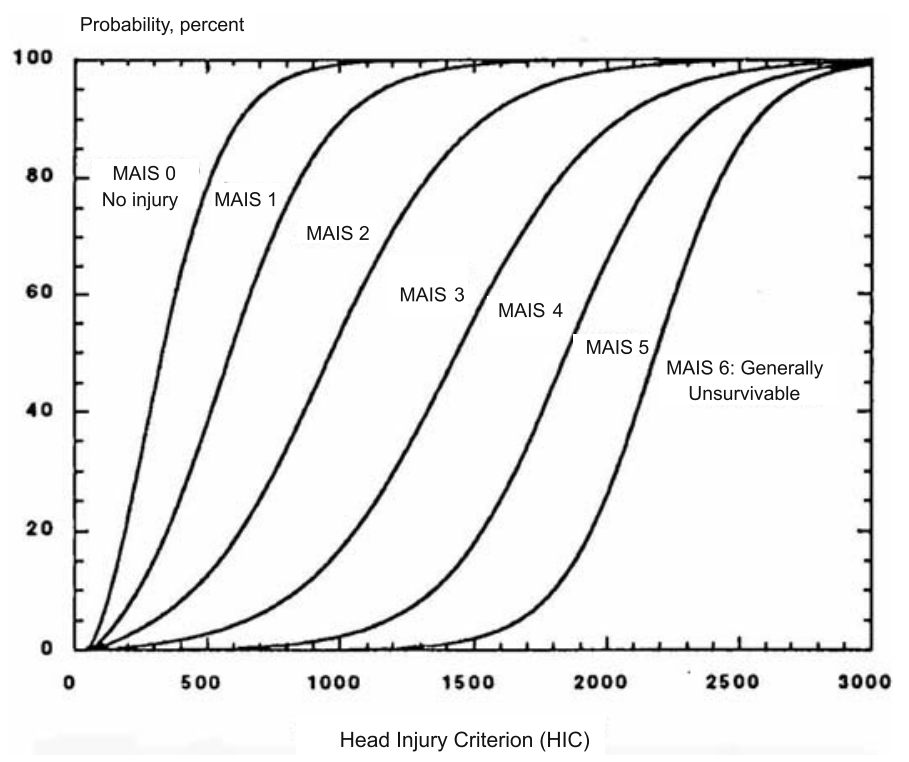

Figure 1. Probability of head injuries of different severities for given HIC values.

dummy in a given crash test do not exceed certain values. This has encouraged a pass/fail process in that if those values are met, then a given car is deemed to be 'safe'.

The reality is that the each injury criterion is merely one data point amongst a family of risk curves, see figure 1 . These data show the probability of injury, to various levels of severity, for given values of the Head Injury Criterion (HIC). The HIC is a variable derived from the acceleration/time history of an accelerometer mounted at the centre of gravity of a dummy's head, when the dummy is exposed to crash forces. The severity of the injuries is assessed by the Abbreviated Injury Scale (AIS). This scale assesses tissue damage and threat to life on a six division ordinal scale running from minor, moderate, serious, severe, critical, to currently untreatable (usually fatal). This scale is now used universally in clinical and research studies as an agreed ranking of the severity of trauma (Gennarelli \& Wodzin 2005). This set of curves, developed by Prasad \& Mertz (1985) shows that an HIC of 1000 is equivalent to an $18 \%$ probability of a severe (AIS 4) head injury, a 55\% probability of a serious (AIS 3) injury and a $90 \%$ probability of a moderate (AIS 2 ) head injury, to the average adult. How those probabilities shift across the spectrum of the population is largely unknown.

Experimental biomechanics has documented the variations across the population for bone strength in particular, and its relationship to bone demineralization and age effects (Pintar et al 1997, Kent et al 2003). As a generalization, fracture tolerance varies by a factor of between 3 and 5 for the strongest and weakest $10 \%$ in the active population exposed on the roads.

For injuries unrelated to fractures, population variation is less well researched. In the absence of actual biomechanical tolerance data on children and small females for example, scaling techniques have been used to produce injury criteria tolerance levels for small females and children (Kleinberger et al 1998). Geometrical and material properties (length, mass, modulus, etc.) are used to scale down to the various smaller sizes from the 50 percentile adult male. Such techniques involving mathematical procedures and 'engineering judgement', have allowed progress to be made in specifying the requirements of advanced restraint systems. 
However, their relationship to the real populations at risk is still to be tested. Accident investigation studies over time will provide some evaluation of the validity of such techniques.

For the brain, abdominal organs, the neck, and soft tissue muscle and skin trauma there is little documentation of actual population variations. Females are shown to be more susceptible to soft tissue neck injury. Skin in older people is somewhat more resistant to threshold lacerations, perhaps a unique biomechanical case of the advantages of growing old.

The injury outcome from a traffic crash is the result of combinations of many factors, both intrinsic and extrinsic to the casualty, and permanent or temporary in nature. For a car occupant, these can be categorised within the three parts of a crash; the pre-crash, crash and post-crash phases, as follows:

Pre-crash variables: Sex, age, height, weight, Body Mass Index (BMI), pre-existing medical conditions, biomechanical tolerance, muscle tone, stomach contents, bladder volume, alcohol, drugs, clothing, seat position in the vehicle, sitting posture, belt position, pre-impact braking.

Crash variables: Impact direction, velocity change, peak vehicle deceleration, crash pulse duration, peak belt load, posture at peak belt load, airbag interactions, point in the cycle of the heart, loads and durations of localized contacts, presence of rear loading, interaction with other occupants.

Post-crash variables: Severity of injuries, combinations of injuries, response time of the emergency services, quality of diagnosis of injuries, quality of treatment, resulting disabilities.

Given the number of such variables it is perhaps surprising that relatively crude accident data, which post facto can only address a small number of these variables, can detect trends at all. Age and sex for example are both sensitive to crash severity. Crash severity is assessed by measuring vehicle damage and relating the amount of crush to the properties of the structure as assessed in experimental collisions involving the make and model of vehicle. See figures 2 and 3 (Mackay et al 1997).

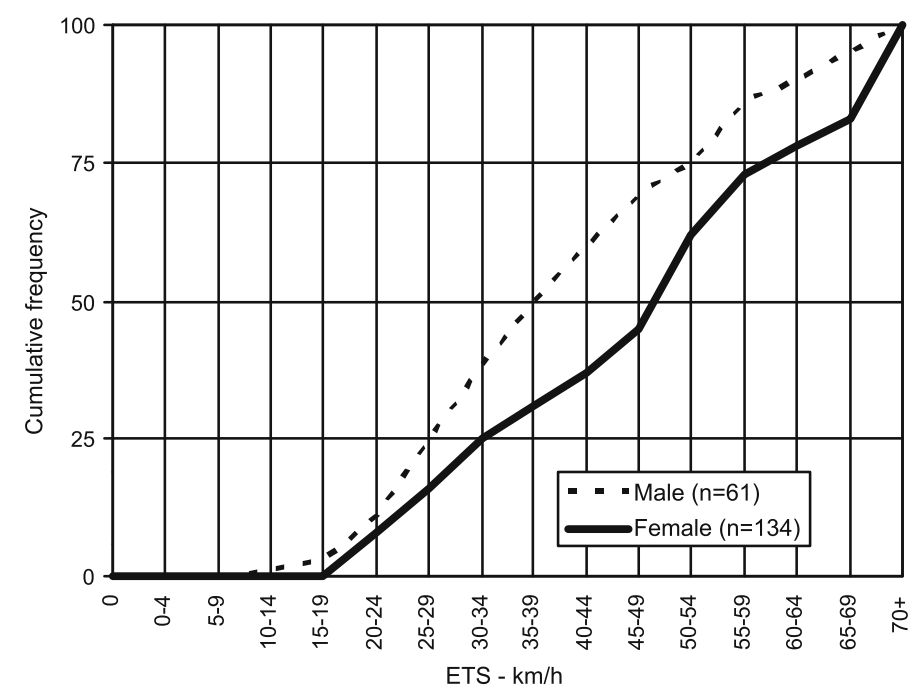

Figure 2. Cumulative frequency curves for restrained front seat occupants receiving AIS $2+$ injuries in frontal collisions, by change in velocity, for males and females (Source: CCIS Study 1983-1992). 


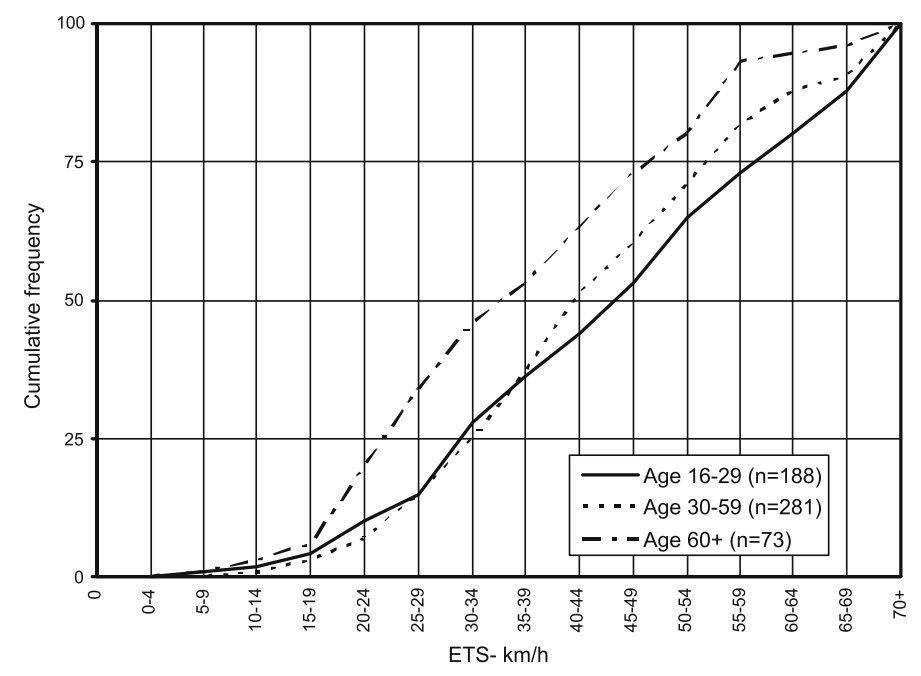

Figure 3. Cumulative frequency curves for restrained front seat occupants receiving AIS 2+ injuries in frontal collisions, by change in velocity, for three age groups (Source: CCIS study 1983-1922).

These data illustrate that the older age groups, particularly those over 60 years of age, and females, are in collisions some $10 \mathrm{~km} / \mathrm{h}$ less in terms of change in velocity than the younger age groups. About the same difference exists between females and male across the whole age range. Expressed differently, males and the young, require over $30 \%$ more kinetic energy than females and those over 60 years of age, to produce the same injury outcomes in say a $40 \mathrm{~km} / \mathrm{h}$ collision.

However, there are probably subtle combinations of some of the other factors listed above which lead to especially high or low levels of risk. Small female drivers currently have to sit close to the steering wheel, and thus have been found in field studies to be at higher risk of airbag related injuries. Tall, thin males have been found to have a greater number of belt related chest injuries than smaller males, probably because the path of the shoulder belt is lower across the rib cage and is thus loading the lower ribs, which fracture more easily than those higher in the rib cage where the rib attachments to the sternum and spine are more substantial and less cartilaginous (Hill et al 1994).

\section{Future trends in biomechanics}

Computer modelling of the human frame now offers the promise of a more precise replication of actual human tissue than does the physical dummy. For example, skull and brain tissue geometrical and engineering properties are so well understood that FEM models of the head can be loaded dynamically to show zones of maximum strain, replicating areas of neurological damage seen in actual brain injured patients (Yao et al 2005). New criteria will be defined based on the position and amount of such zones where the amounts of elongation of the neurons exceed tolerable amounts. Such criteria will replace the overly simplistic values of linear acceleration as measured at the centre of gravity of a physical dummy's head.

Similar models are being developed for the thorax, neck and other body regions, with the ultimate promise of a whole body FEM version of the human frame. However, understanding 


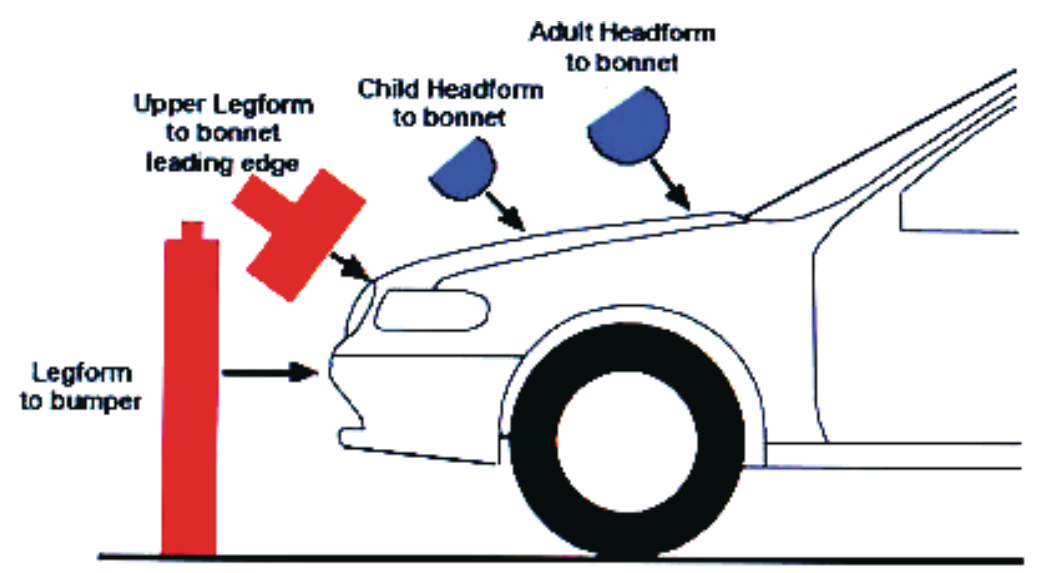

Figure 4. European pedestrian impact test requirements.

the details of the material and the geometrical properties of biological materials and their rate dependency will limit the accuracy of these techniques for some time.

Detailed field accident investigations will become more closely linked to experimental biomechanics both as a way of improving these advanced models of the human frame, and examining the population variations discussed above.

Beyond that, biomechanics at the cellular level will become more important with a greater understanding of the biochemical response to trauma. There are even some indications of genetic variations in susceptibility to impact injuries (Gennarelli et al 2003). Thus crash injury biomechanics will increasingly influence the characteristics of the vehicles on our roads and the ways in which we view and treat traffic accident victims.

Perhaps the most immediate problem area is the application of impact biomechanics to the vulnerable road users. On a global scale, pedestrians, cyclists and motorcyclists outnumber vehicle occupants by four to one. Yet, until recently comparatively little research into the crash phase of such traffic accidents has been conducted. Detailed crash investigations at the scene conducted in the early 1980s demonstrated that for all injury severities the numbers of injuries were equally balanced between vehicle contacts and injuries arising from landing on the ground. For the more serious and life-threatening injuries however, the great majority arise from the primary impact with the front of the striking vehicle (Ashton \& Mackay 1983). More recent work by Kallina (2002) suggests that car exterior design has become less hostile, partly because of design changes for aerodynamic reasons which aim to produce a smooth, rounded front profile, but also because of proposed legislation which specifies the compliance properties of the various front sections of the car (figure 4).

A European Directive is being enacted which specifies the biomechanical responses for various impact tests into the bumper, the bonnet leading edge and the bonnet. These subsystem tests attempt to regulate the engineering properties of those structures to minimize their injury potential. So far this approach only applies to passenger cars but clearly a similar approach could be taken to buses, vans and trucks. More imaginatively, there are engineering proposals which sense the proximity of a pedestrian at appropriate closing speeds and either inflate external airbags or raise the bonnet some $5-8 \mathrm{~cm}$, thus providing a greater ride-down distance for the head contact by a pedestrian.

With the rapid growth of the vehicle population which is occurring in India and elsewhere, these biomechanical benefits need to be introduced into the vehicle fleet as quickly as possible. 
There are other counter-measures which will help in diminishing the risks to the vulnerable road users, mainly in terms of road layout and traffic management, but these are expensive and therefore difficult to implement widely in the short term. Vehicle design can be changed relatively quickly and hence improving exterior design of all vehicles is of high priority. At current rates in many cities in Asia, if the working life of a vehicle is taken as 15 years, then every car over its working life will kill or seriously injure someone. Perhaps this emphasises how vehicle exterior design to minimize injuries to the vulnerable road user is central to the concept of today's cars.

\section{References}

Aldman B 1962 Biodynamic studies of impact protection Acta. Physiol. Scand. 56: 192

Ashton S J and Mackay G M 1983 Benefits from changes in vehicle exterior design - field accident and experimental work in Europe S.A.E. Trans. 830626, 255-263

Cairns H 1946 Crash helmets Br. Med. J. 4470, 322

DeHaven H 1942 Mechanical analysis of survival in falls from heights of fifty to one hundred and fifty feet. War Medicine 586-596

Gennarelli T A, Pintar F A and Yoganandan N 2003 Biomechanical tolerances for diffuse injury and a hypothesis for genotypic variability in response to trauma Proc. AAAM Conf. American Assoc for Automotive Medicine, Chicago 624-628

Gennarelli T A and Wodzin E 2005 The Abbreviated injury scale 2005 American Assoc. Automotive Medicine, Chicago

Gissane W and Bull J P 1962 Injuries from road accidents Practitioner 1988, 489-497

Gurdjian E S and Lissner H R 1958 Head injuries - mechanisms, diagnosis and management. Little Brown \& Co. Boston

Haddon W 1968 The changing approach to the epidemiology, prevention and amelioration of trauma Am. J. Public Health 58: 1431-1438

Hill J, Mackay G M and Morris A 1994 Chest and abdominal injuries caused by seat belt loading, Accident Analysis and Prevention 26(1): 11-26

Huelke D F, Gikas P W and Hendrix R C 1963 Patterns of injury in fatal automobile accidents Proc 6th Stapp Conf. University Minnesota 44-58

Jacobs G, Aeron-Thomas A and Astrop A 2000 Estimating global road fatalities. TRL Report 445 Crowthorne Berks UK

Kallina I 2002 Pedestrian protection — looking for potentials Proc. IRCOBI Conf. Munich 1-18

Kent R, Patrie J, Poteau F, Matsuoka F and Mullen C 2003 Development of an age dependent thoracic injury criterion for frontal impact restraint loading Proc. Enhanced Safety Vehicle Conf. Tokyo

Kleinberger M, Sun E, Eppinger R, Kuppa S and Saul R 1998 Development of improved injury criteria for the assessment of advanced restraint systems, NHTSA, Washington DC

Krug E 2000 (Ed) Injury: A Leading Cause of the Global Burden of Disease WHO Report, Dept. Violence \& Injury Prevention, WHO Geneva

Mackay G M 1969 Some collision aspects of British road accidents Auto. Eng. 59: 500-506

Mackay G M 2005 Quirks of mass accident data bases J. Traffic Injury Prevention 6(4): 299-303

Mackay G M, Ashton S J, Galer M and Thomas P D 1985 Methodology of in-depth studies of car crashes in Britain, S.A.E. No. 850556, 365-390

Mackay G M, Hassan A and Hill J 1997 Adaptive Restraints - their characteristics and benefits, Proc. of Automotive Environmental Impact and Safety Conf. Inst. Mech. Eng., London, 37-52

Melvin J, Baron K J, Little, W C and Gideon T 1998 Biomechanical analysis of Indy race car crashes, Proc. 42nd Stapp Conf, Tempe, Arizona, 247-266

Pintar F A, Yoganandan N, Hines M, Maltese M, McFadden J, Saul R, Eppinger R, Khaewpong N and Kleinberger M 1997 Chest band analysis of human tolerance in side impact. Proc. 41st Stapp Conf. Buena Vista, Florida, 63-74 
Prasad P and Mertz H J, 1985 The position of the United States delegation to the ISO working group 6 on the Use of HIC in the automotive environment. S.A.E. Government Industry Meeting, Washington DC, SAE 85124

Severy D M and Mathewson J H 1954 Automobile barrier impacts Nat. Res. Council Pub. 334: 39

Sheldon J H 1960 On the natural history of falls in old age Br. Med. J. 10: 1685-89

Snyder R G, Foust D R and Dowman B M 1977 Study of impact tolerance through free-fall investigation Uni. Michigan HSRI Report 77

Stapp J P 1951 Human exposure to linear acceleration Aero. Med. Lab. Air Force Report 5912, 2

Thibault L E and Gennarelli T A 1985 Biomechanics of diffuse brain injuries Proc. 10th Exp. Safety Vehicle Conf. NHTSA Washington DC 79-85

Viano D C and Lau IV 1985 Thoracic impact: a viscous criterion Proc. 10th Exp. Safety Vehicle Conf. NHTSA Washington DC 104-113

World Health Organisation 2004 World report on road traffic injury prevention. WHO Geneva

Yao J, Yang J, Otte D and Fredriksson 2005 Reconstruction of head to bonnet top impact in a child pedestrian to passenger car crash, Proc. IRCOBI Conf. Prague CZ 29-40 